This document is confidential and is proprietary to the American Chemical Society and its authors. Do not copy or disclose without written permission. If you have received this item in error, notify the sender and delete all copies.

\title{
Assessing treatment response and prognosis by serum and tissue metabolomics in breast cancer patients.
}

\begin{tabular}{|r|l|}
\hline Journal: & Journal of Proteome Research \\
\hline Manuscript ID & pr-2019-00316w.R1 \\
\hline Manuscript Type: & Article \\
\hline Author: & n/a \\
\hline Complete List of Authors: & $\begin{array}{l}\text { Debik, Julia; Norwegian University of Science and Technology, } \\
\text { Department of Circulation and Medical Imaging } \\
\text { Euceda, Leslie; CAMO Analytics } \\
\text { Lundgren, Steinar; Norges teknisk-naturvitenskapelige universitet, } \\
\text { Department of Circulation and Medical Imaging; St. Olavs Hospital, } \\
\text { Department of Oncology } \\
\text { Gythfeldt, Hedda; Oslo University Hospital, Department of Oncology and } \\
\text { Department of Tumor Biology } \\
\text { Garred, Øystein; University of Oslo, Department of Pathology } \\
\text { Borgen, Elin; Oslo University Hospital, Department of Pathology } \\
\text { Engebraaten, Olav; University of Oslo, Department of Clinical Medicine; } \\
\text { Oslo Universitetssykehus, Department of Oncology and Department of } \\
\text { Tumor Biology } \\
\text { Bathen, Tone; Norges teknisk-naturvitenskapelige universitet, } \\
\text { Department of Circulation and Medical Imaging } \\
\text { Giskeødegărd, Guro; Norges teknisk-naturvitenskapelige universitet, } \\
\text { Department of Circulation and Medical Imaging }\end{array}$ \\
\hline
\end{tabular}

\section{SCHOLARONE \\ Manuscripts}




\title{
Assessing treatment response and prognosis by serum and tissue metabolomics in breast cancer
}

\author{
patients.
}

Julia Debik ${ }^{1 *}$, Leslie R. Euceda',2, Steinar Lundgren3,4, Hedda von der Lippe Gythfeldt ${ }^{5}$, Øystein Garred7, Elin Borgen7,, Olav Engebraaten ${ }^{5,6}$, Tone F. Bathen ${ }^{1}$, Guro F. Giskeødegård ${ }^{*}$

${ }^{1}$ Department of Circulation and Medical Imaging - MR Center, Faculty of Medicine and Health Sciences, NTNU - Norwegian University of Science and Technology, Trondheim, Norway.

${ }^{2}$ CAMO Analytics, Oslo, Norway.

${ }^{3}$ Department of Oncology, St. Olav's University Hospital, Trondheim, Norway. 
${ }^{4}$ Department of Clinical and Molecular Medicine, Faculty of Medicine and Health

Sciences, NTNU - Norwegian University of Science and Technology, Trondheim,

Norway.

${ }^{5}$ Department of Oncology and Department of Tumor Biology, Oslo University Hospital,

Oslo, Norway.

${ }^{6}$ Department of Clinical Medicine, University of Oslo, Oslo, Norway.

${ }^{7}$ Department of Pathology, Oslo University Hospital, Oslo, Norway

AUTHOR ADDRESS: Department of Circulation and Medical Imaging, Faculty of

Medicine and Health Sciences, NTNU, Postbox 8905, 7491 Trondheim, Norway.

\section{Corresponding Authors}

*Guro F. Giskeødegård, Email: guro.giskeødegård@ntnu.no

*Julia Debik, Email: julia.b.debik@ntnu.no 


\begin{abstract}
Locally advanced breast cancer patients have a worse prognosis compared to patients with localized tumors and require neoadjuvant treatment before surgery. The aim of this study was to characterize the systemic metabolic effect of neoadjuvant chemotherapy in patients with large primary breast cancers, and to relate these changes to treatment response and long-term survival.
\end{abstract}

This study included 132 patients with large primary breast tumors randomized to receive neoadjuvant chemotherapy with or without the addition of the antiangiogenic drug bevacizumab. Tumor biopsies and serum were collected before and during treatment; serum additionally six weeks after surgery. Samples were analyzed by nuclear magnetic resonance spectroscopy (NMR).

Correlation analysis showed low correlations between metabolites measured in cancer tissue and serum. Multilevel partial least squares discriminant analysis (PLS-DA) showed clear changes in serum metabolite levels during treatment ( $p$-values $\leq 0.001$ ), including 
unfavorable changes in lipid levels. PLS-DA revealed metabolic differences between tissue samples from survivors and non-survivors collected 12 weeks into treatment with an accuracy of $72 \%(p$-value $=0.005)$, however this was not evident in serum samples.

Our results demonstrate a potential clinical application for serum-metabolomics for patient-monitoring during and after treatment, and indicate potential for tissue NMR spectroscopy for predicting patient survival.

KEYWORDS: Metabolomics, breast cancer, serum, tissue, NMR, response, survival 


\section{INTRODUCTION}

Breast cancer $(\mathrm{BC})$ is the most frequent cancer type in women in Norway. Compared to cancer free women of the same age, five-year survival of BC patients is $90 \%$ in Norway, but ranges from $28-100 \%$, depending on the stage of the disease at the beginning of treatment. ${ }^{1}$ It is however challenging to accurately predict outcome for individual patients, as there is high diversity in prognosis and response to treatment. This is due to the heterogeneous biology of the disease, resulting in patients with similar histology, clinical diagnosis and stage of disease having a different prognosis. ${ }^{2,3} \mathrm{BC}$ is often divided into five genetic intrinsic subtypes, however many studies have shown that there are many subgroups within these groups. ${ }^{4-6}$ One type of treatment will thus not be beneficial for all patients and stratification of patients followed by application of targeted therapy may improve the overall long-term outcome of BC patients.

Locally advanced breast cancer ( $L A B C)$ patients, that is patients with large tumors or extension to lymph nodes, constitutes $10-15 \%$ of diagnosed patients with a higher risk of future metastasis. ${ }^{7}$ Neoadjuvant chemotherapy (NAC) is administered routinely in 
LABC patients. This treatment was initially developed to reduce the size of inoperable tumors prior to surgery and for eradication of potential micrometastasis, but is now also a tool to enable breast-conserving surgery. 8,9

Angiogenesis, the formation of new blood vessels from existing vasculature, has an essential role for supplying nutrients and oxygen to rapidly growing tumors. ${ }^{10}$ This process can be therapeutically targeted by anti-angiogenetic treatment. ${ }^{11}$ Bevacizumab has the ability to inhibit the proangiogenetic vascular endothelial growth factor. ${ }^{12}$ Due to improvements in treatment together with earlier diagnosis, mortality due to BC has decreased during the last years. ${ }^{1}$ However, despite intensive treatment regimes, a great proportion of LABC patients will develop metastatic disease. ${ }^{13,14}$ Additionally, treatment may induce unwanted long-term side effects, such as fatigue, increased risk of cardiovascular diseases (CVD's) and cardiotoxicity. ${ }^{15-19}$ Characterizing the systemic effect of cancer treatment may further enhance our understanding of unwanted sideeffects and potentially identify mechanisms to prevent late effects. 
Metabolomics is a rapidly growing field in medical research, and makes it possible to look at the contents of a biological matrix at the molecular level. Metabolites are downstream biochemical products in the omics cascade, and altered metabolism has been defined as a hallmark of cancer. ${ }^{11}$ Following a minimal sample preparation, a wide range of metabolites can be detected within a short amount of time using nuclear magnetic resonance (NMR) spectroscopy. ${ }^{20}$ NMR metabolomics has already shown potential in stratification of $B C$ patients with respect to treatment response and longterm survival. ${ }^{21,22}$ Most studies so far have focused on metabolomics of invasive tissue biopsies. $^{3,21-24}$ Metabolomics of biofluids is minimally-invasive and repeated sampling is simple. A recent review concludes that many studies have shown impressive associations between biofluid metabolomics and cancer progression, suggesting that NMR metabolomics can be used to provide information with prognostic or predictive value. ${ }^{25}$

The NeoAva study is a phase II randomized clinical trial assessing the effect of antiangiogenesis treatment by bevacizumab in combination with standard NAC. We have 
previously shown that metabolic profiling of tumor tissue by MR spectroscopy has a potential in predicting treatment response in this cohort. ${ }^{21}$ Further, both clinical and gene expression response was shown to differ between patients receiving combination therapy with bevacizumab and chemotherapy alone, and circulating cytokine profiles were found to correlate with different immune cell types at the tumor site. ${ }^{26-28}$ In this study, we performed metabolic profiling of serum samples from patients in the NeoAva study. The main aim was to characterize systemic metabolic effects of NAC in $\mathrm{BC}$ patients, and to relate these changes to treatment response and long-term survival. Additionally, the metabolic information in serum and tissue samples from the same patients were compared, allowing for a better understanding of the difference in their metabolic information. 


\section{MATERIALS AND METHODS \\ Patient and tumor characteristics}

Details of the inclusion criteria are fully described elsewhere. ${ }^{21}$ Briefly, 132 women of age $\geq 18$ years with large $(\geq 2.5 \mathrm{~cm})$, non-metastatic, human epidermal growth factor receptor 2 (HER 2) negative tumors were recruited in the period November 2008-July 2012 in Norway. The study was approved for all centers involved by the Regional Ethics Committee (Approval number S-08354a) and the Norwegian Medical Agency and an informed written consent was obtained from all patients. All patients included in this study received NAC in the form of FEC100 (5-fluorouracil $600 \mathrm{mg} / \mathrm{m}^{2}$, epirubicine 100 $\mathrm{mg} / \mathrm{m}^{2}$, and cyclophosphamide $600 \mathrm{mg} / \mathrm{m}^{2}$ ) followed by taxane-based therapy for 12 weeks, while they were randomized to receive bevacizumab or not. Tissue samples were obtained by ultrasound-guided needle biopsies prior to treatment (TP1) and 12 weeks into treatment (TP2), while surgical biopsies were obtained from the surgically removed tumor (TP3). Non-fasting serum was sampled at TP1, TP2 and TP3, in addition to 6 weeks after surgery (TP4). See Figure S1 for a graphical representation of 
the study design. The study cohort for further analyses has been restricted to contain subjects with full clinical data and available sample material from at least one sampling time point, giving $\mathrm{N}=118$ subjects. In total 357 serum samples and 270 tissue samples were analyzed. Details on the patient and tumor characteristics are summarized in Table 1, while sample availability, including survival data, for each time point is illustrated in Figure S2. 
Table 1. Patient cohort and tumor characteristics

\begin{tabular}{|c|c|c|}
\hline & Survivors $\geq 5$ years & Non-survivors \\
\hline $\mathrm{N}$ & 105 & 13 \\
\hline \multicolumn{3}{|l|}{ Age (years) } \\
\hline Mean (range) & $49.3(25-70)$ & $45.7(31-55)$ \\
\hline \multicolumn{3}{|l|}{ Treatment } \\
\hline Bev + Chemo & 53 & 7 \\
\hline Chemo only & 52 & 6 \\
\hline \multicolumn{3}{|l|}{ RCB class } \\
\hline 0 & 19 & 1 \\
\hline I & 13 & 1 \\
\hline II & 58 & 8 \\
\hline III & 15 & 3 \\
\hline \multicolumn{3}{|l|}{ ER status } \\
\hline Positive & 90 & 10 \\
\hline Negative & 15 & 3 \\
\hline \multicolumn{3}{|l|}{ PgR status } \\
\hline Positive & 62 & 6 \\
\hline Negative & 43 & 7 \\
\hline \multicolumn{3}{|l|}{ Histology } \\
\hline Ductal & 84 & 11 \\
\hline Lobular & 19 & 1 \\
\hline Other & 2 & 1 \\
\hline \multicolumn{3}{|c|}{ Metastasis during follow-up } \\
\hline Yes & 5 & 13 \\
\hline No & 100 & 0 \\
\hline
\end{tabular}

Sample availability varied for each time point, giving a slightly different number of survivors and non-survivors used in the prediction models. Details on sample availability are illustrated in Figure S2. Survivors are patients alive 5 years after treatment start; Bev 
+ Chemo: Bevacizumab treated in addition to neoadjuvant chemotherapy; Chemo only: Chemotherapy only, no bevacizumab; RCB: Residual cancer burden; ER: Estrogen receptor; PgR: progesterone receptor 


\section{Prognostic measures and survival evaluation}

Residual cancer burden (RCB) is a measurement of patient response to NAC. It is a continuous index, which combines pathologic measurements of the primary tumor (size and cellularity) and nodal metastases (number and size). ${ }^{29} \mathrm{RCB}$ can be divided into four classes, where class 0 is equivalent to pathologic complete response (pCR), meaning that no cancer cells are present after treatment.

Patients deceased within 5 years after diagnosis were classified as non-survivors whereas patients surviving $\geq 5$ years were classified as survivors.

\section{NMR experiments and data preprocessing}

Analysis and preprocessing of serum samples

NMR spectra were obtained on a Bruker Avance III Ultrashield Plus spectrometer operating at $600 \mathrm{MHz}$ (Bruker BioSpin $\mathrm{GmbH}$, Rheinstetten, Germany) equipped with a $5 \mathrm{~mm} \mathrm{QCI}$ Cryoprobe. The serum samples were thawed at $4^{\circ} \mathrm{C}$ prior to the analysis. 150 $\mu \mathrm{l}$ of serum was gently mixed with $150 \mu \mathrm{l}$ of buffer $\left(\mathrm{D}_{2} \mathrm{O}\right.$ with $0.075 \mathrm{mM} \mathrm{Na}_{2} \mathrm{HPO}_{4}, 5 \mathrm{mM}$ 
$\mathrm{NaN}_{3}, 3,5 \mathrm{mM}$ TSP, $\mathrm{pH}$ 7.4). The samples were analyzed in 3-mm NMR-tubes. Data acquisition and sample handling was fully automated using a SampleJet with Icon-NMR on TopSpin 3.1 (Bruker BioSpin). Carr-Purcell-Meiboom-Gill (CPMG) spectra with water pre-saturation were acquired at a temperature of $37^{\circ} \mathrm{C}$. The spectra were Fourier transformed to $128 \mathrm{~K}$ after $0.3 \mathrm{~Hz}$ exponential line broadening.

The spectral data were transferred to Matlab R2017b for preprocessing. ${ }^{30}$ The left peak of the alanine doublet at $1.47 \mathrm{ppm}$ was used as a chemical shift reference. Three spectra were removed from the analysis due to poor water suppression after visual inspection. Spectral peaks were aligned to the peaks of the spectrum with the highest correlation to the other spectra using the function icoshift. ${ }^{31}$ The water region (4.3-5.0 ppm) was removed, and the spectral area between 0.2 and $9.2 \mathrm{ppm}$ was used for further analysis. The NMR signals were assigned to metabolites both using the HMDB database, published literature, and an in-house overview over previously assigned spectral peaks in serum based on 2D HSQC acquisitions, and the STOCSY algorithm. ${ }^{32}$ The spectra were mean-normalized prior to quantification. Quantification was performed 
by integrating the region under each peak, giving the relative amounts of metabolites in each sample. If a metabolite had more than one identifiable peak, the mean value of the multiple peaks were calculated and used for further analysis. Signals from ethanol at 1.17 ppm were removed, resulting in 29 distinct peaks (27 metabolites, and two lipid signals, see Table S1). The lipid signals arise from the methyl $\left(-\mathrm{CH}_{3}\right)$ groups at 0.85 ppm (lipid1) and methylene (- $\left.\mathrm{CH}_{2}-\right)$ groups at $1.57 \mathrm{ppm}$ (lipid2), mainly from triglycerides and esterified cholesterol within the lipoprotein particles. ${ }^{33} \mathrm{~A}$ representative spectrum with annotated metabolite peaks is shown in Figure S3.

As evidenced by very high negative correlations (see Figure $1 \mathrm{~A}$ in the Results section) between the serum metabolites and lipid peaks, including the lipids in the analyses overshadowed changes in the low-molecular weight serum metabolites. We therefore removed the lipid peaks and normalized the metabolites a second time prior to statistical analyses.

Analysis and preprocessing of tissue samples 
A total of 270 tissue samples were analyzed by high resolution (HR) magic angle spinning (MAS) NMR. Details of NMR experiments, preprocessing and quantification of the tissue samples have been described previously. ${ }^{21}$ Briefly, tissue samples (mean weight: $4.1 \mathrm{mg}$ ) were analysed at $5{ }^{\circ} \mathrm{C}$ on a Bruker Avance DRX600 spectrometer equipped with a ${ }^{1} \mathrm{H} /{ }^{13} \mathrm{C}$ MAS rotor. A spin-echo one dimensional experiment with presaturation (cpmgpr1d, Bruker BioSpin, Germany) was recorded for all samples, with effective echo time of $77 \mathrm{~ms}$, a spectral width of $20 \mathrm{ppm}$ ( -5 to $15 \mathrm{ppm})$, and 256 scans.

Spectra were baseline corrected, peak aligned using the icoshift algorithm ${ }^{31}$, and normalized by PQN ${ }^{34}$ after removal of lipid residuals. Quantified metabolites were normalized by PQN.

\title{
Statistical analysis
}

\section{Multivariate analyses}

\author{
All variables were auto-scaled prior to multivariate analyses. Principal component \\ analysis $^{35}$ (PCA) was performed on the quantified serum metabolites as a first step in \\ the exploratory analysis.
}


Partial least squares discriminant analyses (PLS-DA) were employed to fit classification models for different clinical variables. ${ }^{36}$ PLS-DA models were fitted and validated using 10 -fold cross-validation, which was repeated 20 times. The optimal number of latent variables was chosen to be the number of latent variables corresponding to the first minima in the cross-validated classification error. Averaged sensitivities and specificities of the 20 iterations are reported. To verify the statistical significance of the models, permutation testing was employed, where the original class labels were shuffled among the individuals. ${ }^{37}$ New models were fit to these permuted data sets and the classification error was calculated. The proportion of classifications equal to or better than the original classification was used to calculate the p-values. The permutations were repeated 1000 times and $p$-values $\leq 0.05$ were considered significant. For the PLS-DA plots, the $y$ variance was condensed into the first LV through orthogonal projection to latent structures (OPLS-DA) in cases where the optimal model had more than one LV. This orthogonalization does not improve the model accuracy, but rather the model interpretation, as the predictive from non-predictive variation is separated. ${ }^{36}$ 
Metabolomics data is complex and many factors (such as age, disease state and genetics) influence the metabolic profile of a biological sample, thus the variations between samples of different individuals are often higher than the variations within the samples of one individual. Variations, as a result of treatment effect, can be overshadowed by the between-subject variations. The total effect is thus undetectable if the main focus is the average effect. Multilevel PLS-DA is an extension of PLS-DA and consists of two steps. ${ }^{38}$ First, the variation between individuals is separated from the variation within the samples. Second, PLS-DA analysis is performed on the withinsubject variation. This is an effective tool for longitudinal data, where there are two or more multivariate measurements per subject. Since the multilevel PLS-DA models contain multiple measurements for each patient, $10 \%$ of the patients were left out during each iteration, which was repeated 20 times.

PCA, PLS-DA and multilevel PLS-DA analyses were carried out in Matlab R2017b using the PLS Toolbox 8.6.2. ${ }^{39}$ The loading plots of the orthogonalized PLS-DA and multilevel PLS-DA analyses were colored according to the variable (here metabolite) importance 
score (VIP score). The VIP score is a measure of how important each variable was for creating the discrimination model. It is calculated as a weighted sum of squares of the PLS loadings, where the weights are based on the amount of $y$-variance explained in each dimension. ${ }^{40} \mathrm{~A}$ metabolite with a VIP score larger than or equal to 1 was considered to be important in the discrimination model.

\section{Univariate data analysis}

Due to non-normality of the serum metabolites, the non-parametric Wilcoxon-signedrank test was used to test the significance of the changes in serum metabolite levels between time points. ${ }^{41} \mathrm{P}$-values were adjusted using the Benjamini-Hochberg procedure and significance was considered for q-values $\leq 0.05 .{ }^{42}$

In this study, both serum and tumor samples from the same BC patients were analyzed, enabling us to investigate how much of the tissue-metabolic profile is reflected in the serum metabolome. To investigate this, Pearson-correlations between all quantified metabolites in the serum and tissue samples were calculated. P-values for significance were adjusted for multiple comparisons using the Benjamini-Hochberg procedure, and 
significance was considered for $q$-values $\leq 0.05$. The calculations and graphical

representations of the correlation were performed in the R software environment using

the corrplot package. ${ }^{43,44}$

Statistical analyses of serum metabolites were performed on quantified metabolites. For tissue samples, multivariate analyses were performed on the whole NMR spectra as in Euceda et al. ${ }^{21}$ while correlation analysis was performed using quantified metabolite levels. 


\section{RESULTS \\ Correlation analysis of serum and tissue metabolic profiles}

Availability of both tissue biopsies and serum samples from the same BC patients, enabled to investigate how much of the tumor metabolism is reflected in the serum. The majority of the correlations between the serum metabolites were high (Figure 1A). The low-molecular weight serum metabolites had a highly negative correlation with the lipid peaks, while they were positively correlated with each other. There were fewer high correlations between tissue metabolites. However, tissue levels of taurine and glucose, and glutamate and lactate were highly correlated $(\varrho=0.903$ and 0.714 respectively; qvalues $<0.001)$. This figure also shows that correlations between serum and tissue metabolites, although some were significant, were low $(0.005 \leq|\mathrm{Q}| \leq 0.269$; q-values $\leq$ 0.05). Serum lactate was not correlated with tissue lactate $(\rho=0.061$, $q$-value $=0.835)$. In addition, choline stands out from the other tissue metabolites, with low but significant correlations with the majority of the serum metabolites $(0.074 \leq|\mathrm{e}| \leq 0.269)$. To emphasize correlations between the low-molecular weight metabolites, the analyses 


\begin{abstract}
were repeated with the lipid peaks removed. Figure 1B shows correlation analyses of serum and tissue metabolic profiles after the removal of lipid peaks in serum data and a second normalization. The correlations in serum metabolites are then highly affected in both magnitude and direction.
\end{abstract}



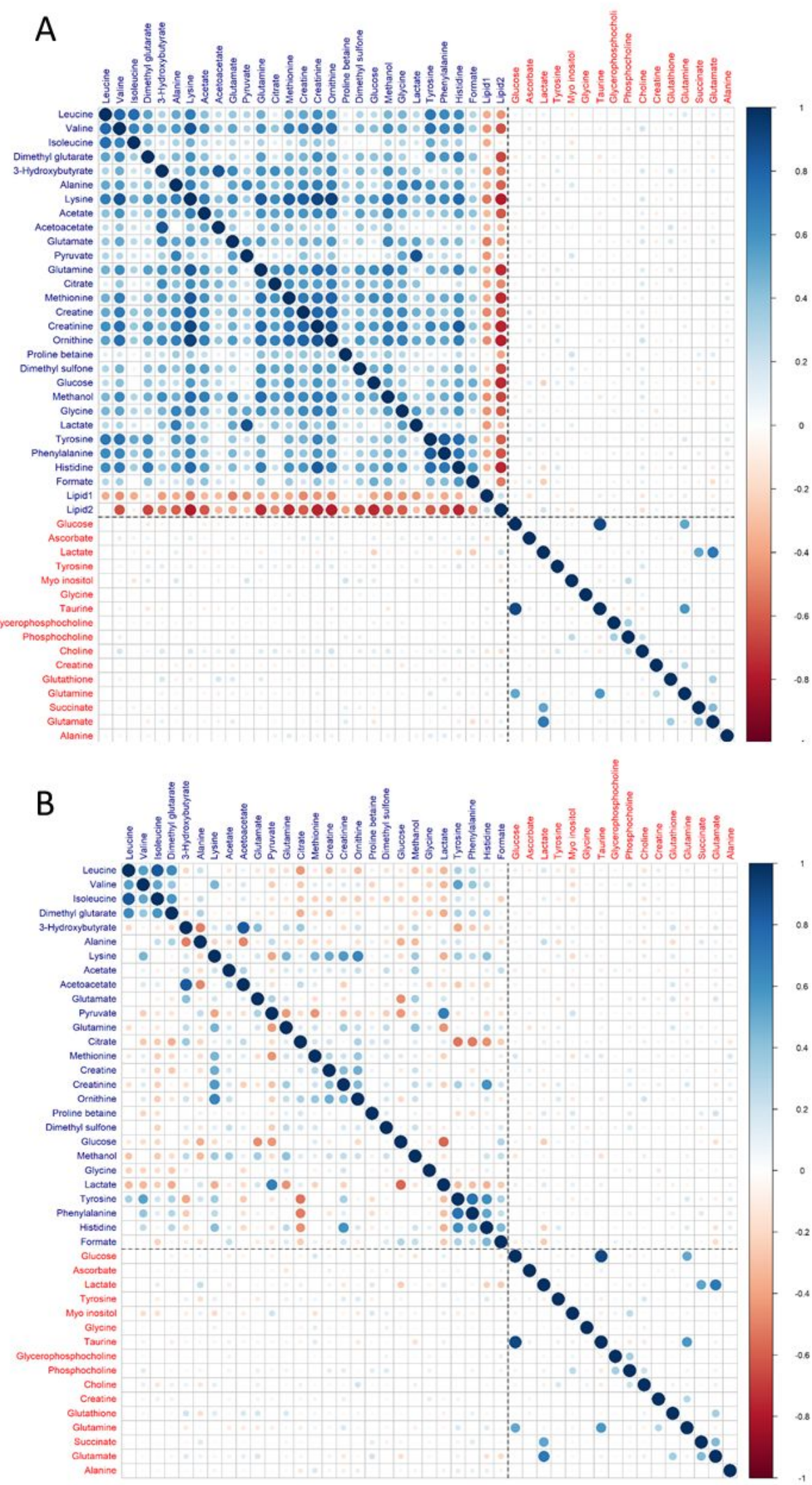

ACS Paragon Plus Environment 
Figure 1. Significant Pearson correlations between metabolites in serum (blue) and tissue (red) samples. A: Whole data set; B: After removal of lipid peaks from serum data and a second normalization. Color intensity and circle sizes are proportional to the correlation coefficients. Red and blue circles indicate negative and positive correlations, respectively. Only patients with both serum and tissue samples available (TP1, TP2 and TP3) have been included in this analysis. 


\section{The effect of neoadjuvant chemotherapy on serum metabolic profiles}

PCA analyses of serum metabolites did not show any clear trend or grouping of the patients with respect to the time point at which the samples were obtained (Figure S4).

However, by employing multilevel PLS-DA and thus removing the between-subject variation in the data, significant changes in the serum metabolic profiles between each time point during treatment were revealed. Table 2 summarizes the fit of the multilevel PLS-DA models on serum data without lipid peaks included. PLS-DA results for separating TP1 and TP2 with and without including the lipids are shown in Figure 2.

First, when the lipid peaks are included in the multilevel analyses, it is clear that the amount of lipids in serum increase during treatment (Figure 2A). The same is evident throughout the treatment period as seen in Figure S5, which shows multilevel analysis comparing TP1 with TP4 when lipids are included. Removal of lipid peaks to emphasize changes within the metabolic profile did not have a significant influence on the prediction accuracy of the models. Further results are derived from the serum metabolic data without including the lipid peaks. 
Scores and loading plots of the multilevel PLS-DA models separating different time points are displayed in Figure S6, where the loadings are colored according to the VIP scores. The most important metabolites in discriminating between serum metabolic profiles at TP1 and TP2 are creatinine $(\downarrow)$, creatine $(\downarrow)$, isoleucine $(\uparrow)$, ornithine $(\downarrow)$ and histidine $(\uparrow)$ (Figure 2B), where the arrow shows the direction of the change with the treatment course. For discriminating TP2 from TP3 creatine $(\uparrow)$, valine $(\uparrow)$, dimethylglutarate $(\downarrow)$ and pyruvate $(\downarrow)$ are of highest importance. Finally, for discriminating between serum metabolic profiles at TP4 and TP3, valine $(\uparrow)$, glycine $(\downarrow)$, dimethylglutarate $(\uparrow)$ and methionine $(\uparrow)$ are the most important metabolites.

Table 2. Summary of multilevel PLS-DA applied on serum metabolites, after the removal of lipid peaks and a second normalization.

\begin{tabular}{l|c|c|c|c}
\hline & No of LV's & Class accuracy (\%) & Sensitivity/Specificity (\%) & P-value \\
\hline TP1 vs TP2 & 4 & 90 & $90 / 90$ & $<0.001$ \\
TP2 vs TP3 & 2 & 77 & $77 / 77$ & $<0.001$ \\
TP3 vs TP4 & 4 & 87 & $87 / 87$ & $<0.001$ \\
\hline
\end{tabular}

Sensitivities and specificities are averaged on 20 repetitions of 10 -fold cross validation. The reported $p$-values are based on permutation testing, with 1000 random permutations of the original class labels. Significant classification models in bold. LV: latent variable. 
The median percentage change of each metabolite level between the different time points is displayed in Table 3, with corresponding q-values to assess statistical significance. Most significant changes occur between TP1 and TP2; however the metabolic profiles change significantly throughout the treatment period. Only two metabolites exhibited significant changes across all sampling time points during the treatment course: dimethylglutarate $(\uparrow \downarrow \uparrow)$ and acetate $(\downarrow \uparrow \uparrow)$. 

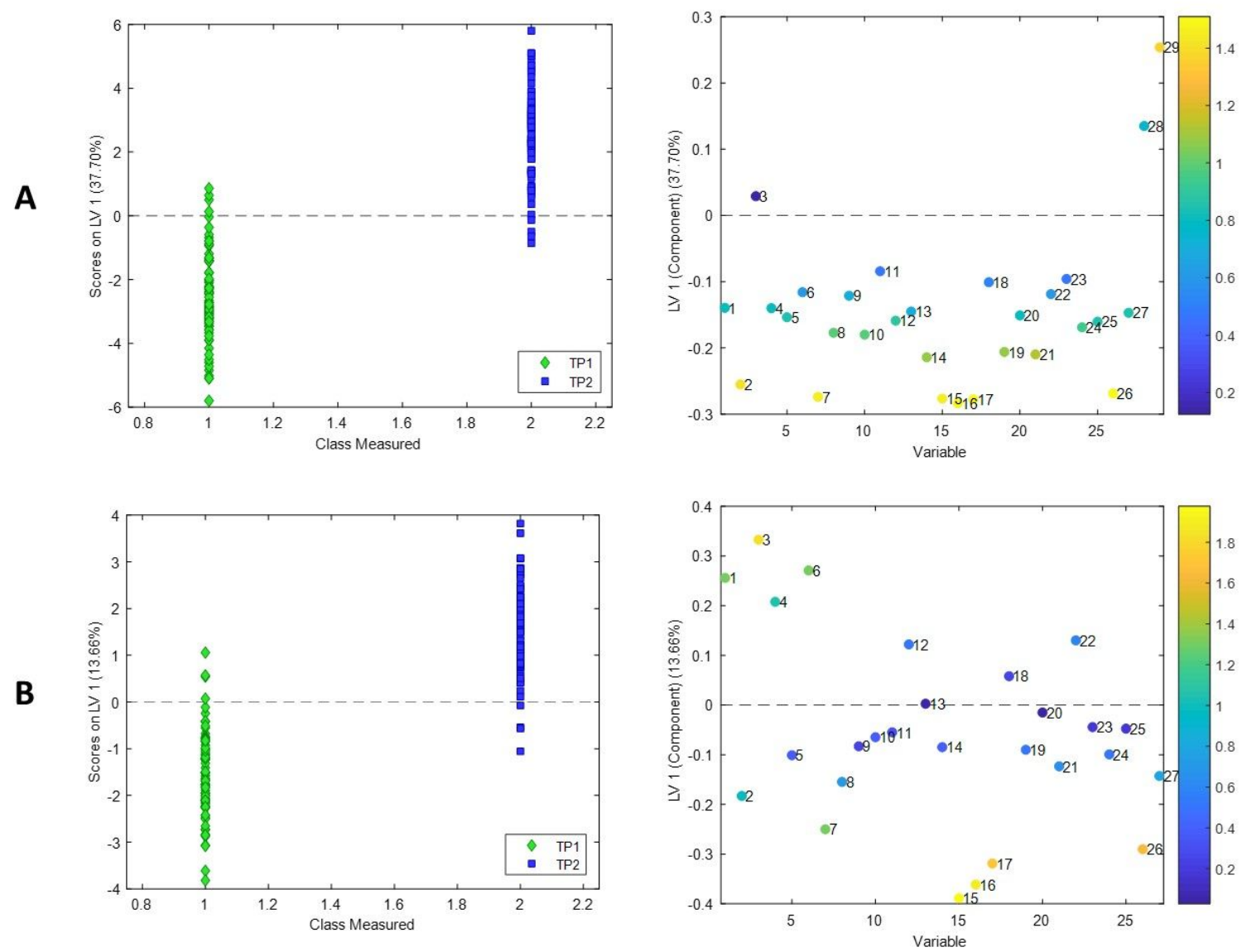

Figure 2. Scores and loadings plots from the multilevel PLS-DA analyses for discriminating between the serum metabolic profiles at TP2 from TP1. A: Analysis including lipid peaks. B: Analysis after excluding lipid peaks and a second normalization. Orthogonalized loadings colored according to VIP scores. LV: latent variable. 1: leucine; 2: valine; 3 : isoleucine; 4: dimethylglutarate; 5 : tri-hydroxybutyrate; 6 : alanine; 7 : Iysine; 8: acetate; 9: acetoacetate; 10: glutamate; 11: pyruvate; 12: glutamine; 13: citrate; 14: 
methionine; 15: creatine; 16: creatinine; 17: ornithine; 18: proline-betaine; 19: dimethylsulfone; 20: glucose; 21: methanol; 22: glycine; 23: lactate; 24: tyrosine; 25: phenylalanine; 26: histidine; 27: formate; 28: lipid1; 29: lipid2.

Table 3. Median percentage changes in the serum metabolite levels during treatment.

\begin{tabular}{|c|c|c|c|c|c|c|c|}
\hline & Metabolite name & $\begin{array}{l}\text { TP1 to } \\
\text { TP2 (\%) }\end{array}$ & q-value & $\begin{array}{c}\text { TP2 to } \\
\text { TP3 (\%) }\end{array}$ & q-value & $\begin{array}{c}\text { TP3 to } \\
\text { TP4 (\%) }\end{array}$ & q-value \\
\hline 1 & Leucine & 4.88 & 0.001 & -2.52 & 0.613 & 4.64 & 0.029 \\
\hline 2 & Valine & -1.76 & 0.082 & 5.55 & 0.004 & 7.19 & $<0.001$ \\
\hline 3 & Isoleucine & 12.46 & $<0.001$ & -0.95 & 0.706 & -0.73 & 0.512 \\
\hline 4 & Dimethylglutarate & 3.28 & 0.036 & -8.27 & 0.001 & 9.36 & 0.001 \\
\hline 5 & Alanine & -3.26 & 0.294 & 2.06 & 0.386 & 2.48 & 0.271 \\
\hline 6 & Lysine & 5.06 & 0.001 & -4.24 & 0.010 & 1.95 & 0.319 \\
\hline 7 & Acetate & -3.43 & $<0.001$ & 1.81 & 0.010 & 3.57 & 0.002 \\
\hline 8 & Acetoacetate & -2.16 & 0.156 & 5.82 & 0.030 & -0.20 & 0.589 \\
\hline 9 & 3-Hydroxybutyrate & 0.84 & 0.562 & 3.65 & 0.212 & 5.82 & 0.178 \\
\hline 10 & Glutamate & 1.63 & 0.974 & -0.06 & 0.613 & 1.22 & 0.280 \\
\hline 11 & Pyruvate & -3.83 & 0.808 & -6.28 & 0.010 & -0.98 & 0.722 \\
\hline 12 & Glutamine & 1.81 & 0.244 & 0.16 & 0.955 & -2.37 & 0.062 \\
\hline 13 & Citrate & -2.38 & 0.974 & -1.82 & 0.953 & -7.37 & 0.039 \\
\hline 14 & Methionine & -1.83 & 0.294 & 3.40 & 0.187 & 4.80 & 0.002 \\
\hline 15 & Creatine & -13.30 & $<0.001$ & 9.01 & $<0.001$ & 5.34 & 0.089 \\
\hline 16 & Creatinine & -7.80 & $<0.001$ & 2.30 & 0.185 & 4.19 & 0.040 \\
\hline 17 & Ornithine & -6.33 & $<0.001$ & 1.38 & 0.355 & 4.17 & 0.002 \\
\hline 18 & Proline-betaine & -3.10 & 0.974 & 4.22 & 0.585 & 1.58 & 0.604 \\
\hline 19 & Dimethyl-sulfone & -2.14 & 0.294 & 4.66 & 0.207 & 3.31 & 0.163 \\
\hline 20 & Methanol & -2.74 & 0.294 & -0.54 & 0.706 & -1.48 & 0.452 \\
\hline 21 & Glucose & -3.83 & 0.095 & 1.61 & 0.491 & -0.51 & 0.798 \\
\hline 22 & Glycine & 3.08 & 0.156 & 0.81 & 0.603 & -6.08 & 0.001 \\
\hline
\end{tabular}




\begin{tabular}{|c|c|c|c|c|c|c|c|}
\hline 23 & Lactate & -1.19 & 0.974 & -3.01 & 0.813 & -14.40 & 0.002 \\
\hline 24 & Tyrosine & -3.83 & 0.303 & -2.38 & 0.603 & 4.43 & 0.452 \\
\hline 25 & Phenylalanine & -1.50 & 0.887 & 0.83 & 0.799 & 8.24 & 0.025 \\
\hline 26 & Histidine & -9.81 & $<0.001$ & -0.33 & 0.706 & 9.84 & 0.010 \\
\hline 27 & Formate & -10.60 & 0.036 & -6.57 & 0.603 & -5.17 & 0.936 \\
\hline & & & & $>10 \%$ & & $<-10 \%$ & \\
\hline & & & & 5 to $10 \%$ & & -5 to $10 \%$ & \\
\hline & & & & 0 to $5 \%$ & & 0 to $-5 \%$ & \\
\hline
\end{tabular}

Only patients with samples available at each of the two time points were included when calculating the percentage changes. Q-values show $p$-values obtained from Wilcoxon signed-rank test, adjusted for multiple comparisons. Significant changes are marked in bold.

\section{The effect of bevacizumab on serum metabolic profiles}

We further examined if the serum metabolites are affected by treatment with the drug bevacizumab in addition to chemotherapy. A significant discrimination model for separating patients receiving and not receiving bevacizumab was obtained at TP2, but not at later time points (accuracy $=64 \% ; p$-value $=0.014$, Figure $3 \mathrm{~A}$ and Table 4 ), even though the admission of bevacizumab was continued until TP3. The most important metabolites in the discrimination model for TP2 are higher levels of leucine, acetoacetate, tri-hydroxybutyrate and lower of formate (VIP scores 1.76, 1.59, 1.56 and 1.47 
respectively) for the group of patients treated with bevacizumab compared to patients treated with chemotherapy only. 
A
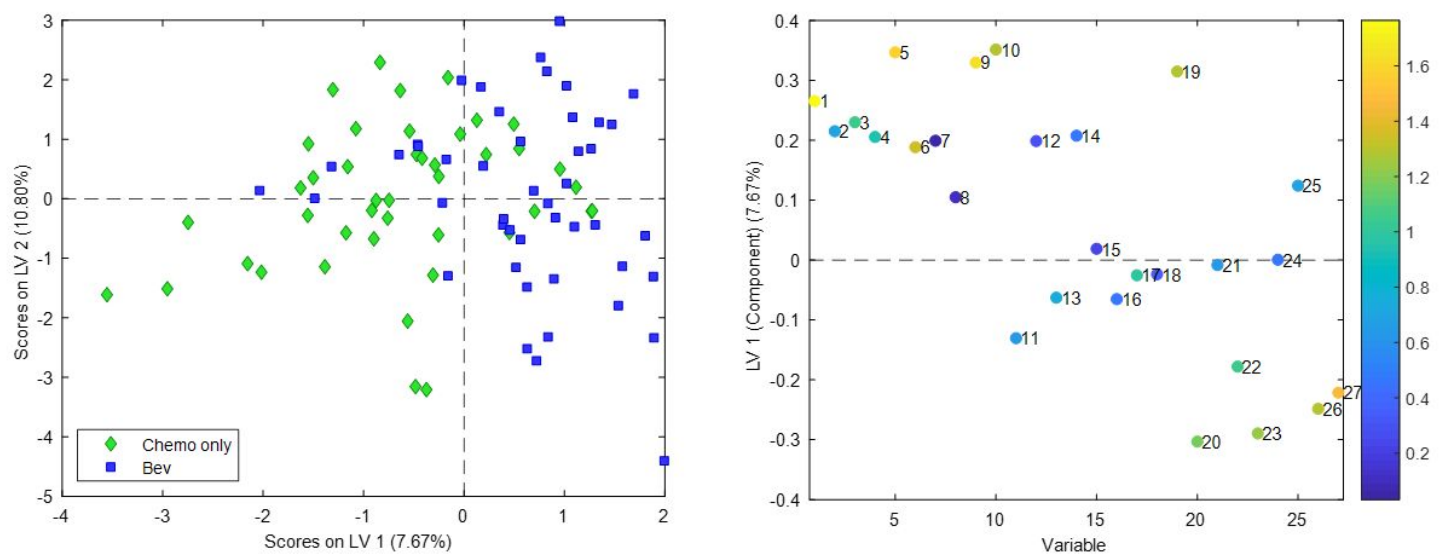

B
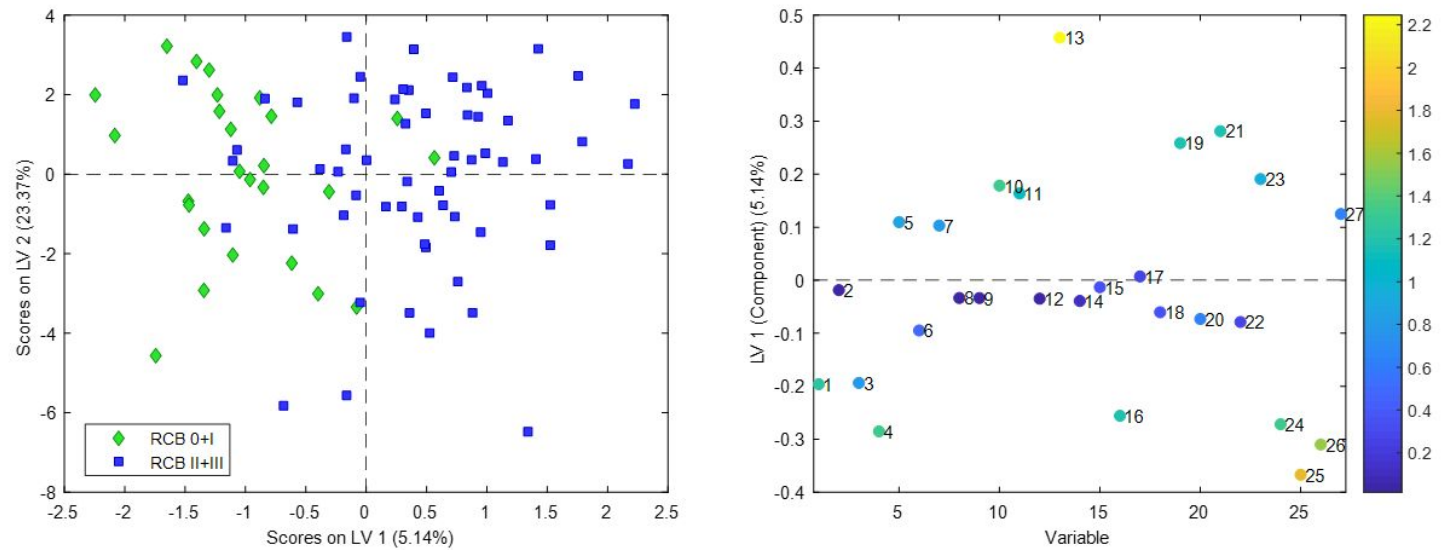

Figure 3. Scores and loading plots of the PLS-DA models for serum metabolic profiles. A:

Bevacizumab-treated vs Chemotherapy only at TP2. B: RCB 0 or I vs RCB II or III at TP4.

Orthogonalized loadings colored according to the VIP scores. 1: leucine; 2: valine; 3 :

isoleucine; 4: dimethylglutarate; 5: tri-hydroxybutyrate; 6: alanine; 7: lysine; 8: acetate; 9:

acetoacetate; 10: glutamate; 11: pyruvate; 12: glutamine; 13: citrate; 14: methionine; 15:

creatine; 16: creatinine; 17: ornithine; 18: proline-betaine; 19: dimethylsulfone; 20: 
glucose; 21: methanol; 22: glycine; 23: lactate; 24: tyrosine; 25: phenylalanine; 26:

histidine; 27: formate. 
Table 4. Summary of PLS-DA classification models fitted to the serum and tissue metabolic profiles at different time points.

\begin{tabular}{|c|c|c|c|c|c|c|}
\hline & $\begin{array}{l}\text { Discriminated } \\
\text { classes }\end{array}$ & $\begin{array}{l}\text { Time } \\
\text { point }\end{array}$ & $n$ & $\begin{array}{c}\text { Class } \\
\text { accuracy (\%) }\end{array}$ & $\begin{array}{c}\text { Sensitivity/ } \\
\text { Specificity (\%) }\end{array}$ & $\begin{array}{c}\text { Permutation } \\
\text { p-value }\end{array}$ \\
\hline \multirow{11}{*}{$\frac{\varepsilon}{\frac{\xi}{2}}$} & \multirow{3}{*}{$\begin{array}{c}\text { Bev-treat. / } \\
\text { Chemo treat. only }\end{array}$} & TP2 & 89 & 64 & $58 / 70$ & 0.0140 \\
\hline & & TP3 & 93 & 59 & $60 / 57$ & 0.0870 \\
\hline & & TP4 & 86 & 57 & $67 / 47$ & 0.0960 \\
\hline & \multirow{4}{*}{$\begin{array}{l}\text { RCB class } 0+I / \\
\text { RCB class II + III }\end{array}$} & TP1 & 89 & 36 & $27 / 44$ & 0.9580 \\
\hline & & TP2 & 89 & 48 & $33 / 63$ & 0.6500 \\
\hline & & TP3 & 93 & 58 & $58 / 57$ & 0.1700 \\
\hline & & TP4 & 86 & 69 & $65 / 73$ & 0.0010 \\
\hline & \multirow{4}{*}{5 year survival } & TP1 & 89 & 37 & $5 / 70$ & 0.7700 \\
\hline & & TP2 & 89 & 64 & $48 / 81$ & 0.2570 \\
\hline & & TP3 & 93 & 61 & $43 / 79$ & 0.1780 \\
\hline & & TP4 & 86 & 48 & $23 / 73$ & 0.5620 \\
\hline \multirow{3}{*}{$\stackrel{0}{\stackrel{0}{n}}$} & \multirow{3}{*}{5 year survival } & TP1 & 105 & 58 & $30 / 86$ & 0.2190 \\
\hline & & TP2 & 78 & 72 & $55 / 90$ & 0.0050 \\
\hline & & TP3 & 87 & 57 & $26 / 88$ & 0.2210 \\
\hline
\end{tabular}

Sensitivities and specificities are averaged on 20 repetitions of 10 -fold cross validation. The reported $p$-values are based on permutation testing, with 1000 random permutations of the original class labels. Significant classification models are marked in bold. n: number of samples included

model. 
Serum metabolic differences between responders and non-responders of neoadjuvant
treatment PLS-DA classification models were fitted to the serum metabolites for each time point separately to examine if there were metabolic differences between patients with a good or poor response to treatment. The model results are summarized in Table 4. Summary of PLS-DA classification models fitted to the serum and tissue metabolic profiles at different time points. Patients with good response (RCB 0 or I) could be significantly discriminated from patients with a poor response (RCB II or III) at TP4 with an accuracy of $69 \%$ ( $p$-value $=0.001$, Figure 3B). The most important metabolites in the discrimination were citrate, phenylalanine and histidine (VIP scores 2.25, 1.75 and 1.53, respectively), with higher levels of citrate and lower of the latter in RCB II or III compared to RCB 0 or I patients.

\section{Predicting survival from serum and tissue metabolic profiles}

Discrimination models were fitted to assess if there is predictive power in the serum and tissue metabolites to predict long-term outcome. Results of the analyses show that the 
serum metabolites have no predictive power for 5-year survival (Table 4). Similar models were employed on the tissue metabolic profiles at the different time points, and showed that tissue metabolic profiles at TP2 have a predictive potential for discriminating survivors from non-survivors, with a prediction accuracy of $72 \%$ ( $p$-value $=0.0050$ ). Scores and loading plots for the corresponding PLS-DA model at TP2 are displayed in Figure 4.
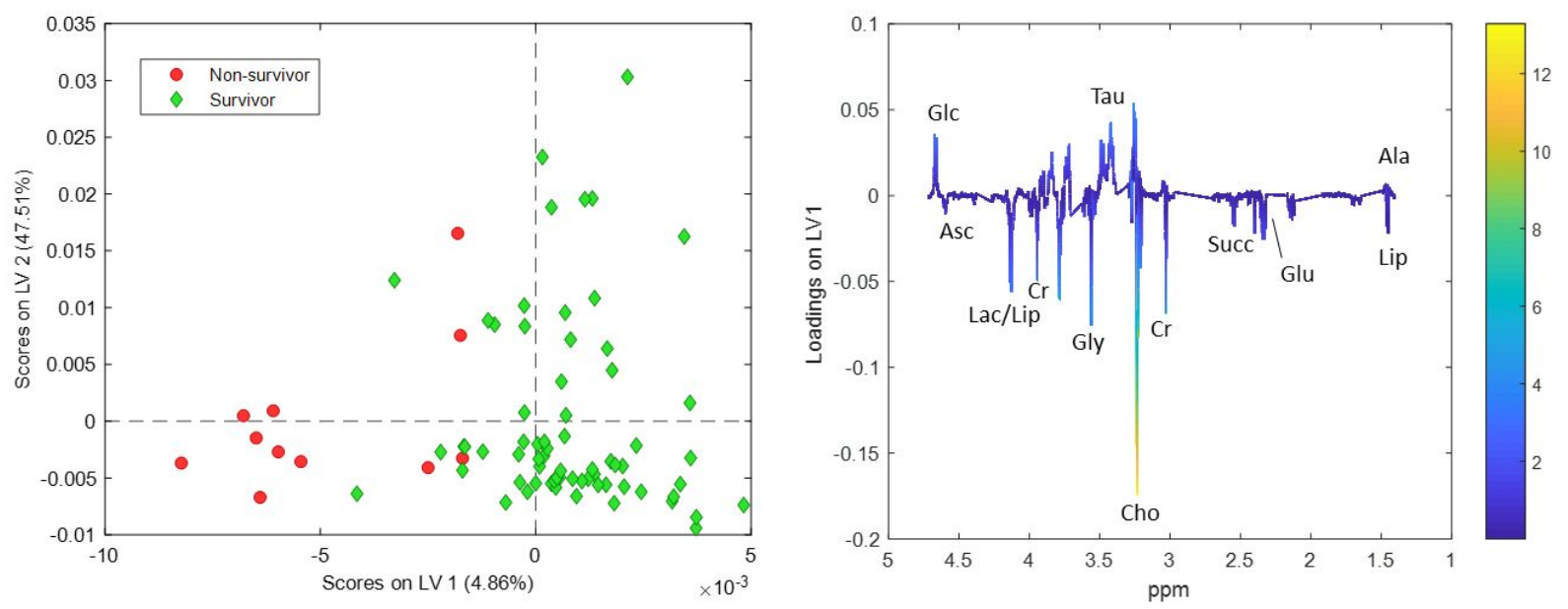

Figure 4. Scores and loadings plots for predicting survival from tissue metabolic profiles at TP2. Orthogonalized loadings colored according to their VIP score. LV: latent variable; Glc: glucose; Asc: ascorbate; Lac: lactate; Lip: lipid; Gly: glycine; Tau: taurine; Cho: 
cholines (glycerophosphocholine, phosphoscholine and choline); Cr: creatine; Succ:

succinate; Glu: glutamate; Ala: alanine; 


\section{DISCUSSION}

In this study we show that the NMR-based metabolic profile of serum from BC patients undergoing NAC changes significantly throughout treatment. Further, we show that 5year survival can be predicted from metabolic profiles in tissue, but not serum. Significant associations between serum metabolic profiles and response to treatment, in addition to changes in the serum metabolic profiles in patients receiving bevacizumab, were detected.

Several factors affect the serum metabolome, such as diet, age, body mass index (BMI), drug use and diurnal variations. ${ }^{45-47}$ The serum metabolome will contain metabolic signals from both the tumor itself and the host organism, both affected by treatment. Some studies have investigated the difference in the serum metabolic profiles of women with BC compared to healthy controls, showing that presence of the tumor has an evident effect on the serum metabolome ${ }^{48-52}$, while only few have looked into treatment-induced changes. ${ }^{53,54}$ A previous study revealed baseline levels of formate and acetate as 
potential predictive biomarkers of treatment response in metastatic BC patients, linking these changes to the accelerated proliferation of aggressive BC cells. ${ }^{55}$ In this study, we describe significant serum metabolic changes in response to treatment at all time points, showing that $B C$ treatment has an effect on the overall metabolism. Particularly lipid levels in serum increased throughout the treatment course (Figure 2A, Figure S5). These results are in agreement with a previous study where we describe serum metabolic changes from adjuvant BC treatment, where unfavorable changes in the lipoprotein profiles were observed during treatment. ${ }^{56}$ Altered lipid metabolism may predispose for weight gain, increased risk of CVD and a worse overall health and quality of life. Increased lipid levels in serum post treatment have additionally been observed and associated with an increased risk of disease recurrence..$^{57}$

The most evident effect of $B C$ treatment on the serum metabolome occurred during the first weeks of treatment (TP1 to 2) and from surgery to 6 weeks follow-up (TP3 to 4). When comparing samples acquired before treatment onset and 12 weeks into treatment, 11 of the 27 metabolites changed significantly, mainly to decreased levels. Comparing 
the first weeks of treatment revealed decreased histidine, creatine, creatinine and ornithine levels and increased isoleucine, to be of highest importance (Figure 2B). Serum levels of isoleucine were previously shown to be upregulated in of metastatic compared to early $\mathrm{BC}^{50,58}$ and higher isoleucine has also been associated with $\mathrm{pCR}^{54}$. Thus the predictive value of changes in isoleucine levels should be further investigated. Creatinine is a breakdown product of phosphocreatine in muscles and is usually produced at a constant rate by the body; it is thus plausible that the observed increase is induced by treatment. Creatine, creatinine and ornithine are amino acids closely linked together through the arginine and proline metabolism pathway, through which glutamate is synthetized from arginine and proline.

Twelve weeks into treatment, increased levels of valine and creatine, and decreased levels of dimethylglutarate, lysine and pyruvate were observed, compared to six weeks into treatment. Similarly, increased levels of valine and creatine during BC treatment, compared to baseline levels, were observed in a longitudinal study with HER-2 positive $\mathrm{BC}$ patients in the trastuzumab and everolimus treatment arm. ${ }^{53}$ Increased valine levels 
have also been shown to be important in discriminating $\mathrm{BC}$ patients from healthy controls (post-treatment). ${ }^{49}$ Pyruvate is a key intermediate in several metabolic pathways throughout the cell, including gluconeogenesis and the Krebs cycle; lower pyruvate levels therefore possibly reflect an increased energy metabolism due to the treatment.

Patients switched from FEC treatment to taxane-based therapy twelve weeks into the treatment (TP2), followed by no further treatment, other than surgery, between the last two sampling points (TP3 to 4). It appears that the serum metabolism tends to return to its pre-treatment state in this period; valine, acetate, creatine, ornithine and histidine all experienced a decrease at the beginning of treatment, followed by an increase after surgery. Glycine levels remained relatively constant throughout treatment, but decreased significantly after treatment. Low levels of circulating glycine have previously been associated with metabolic syndrome; this decrease may thus indicate a negative sideeffect of treatment. ${ }^{59}$ 
Five year survival was predicted with an accuracy of $72 \%$ at TP2. Non-survivors had higher lactate and glycine levels compared to survivors at TP2, which is in accordance with previous studies in similar patient cohorts. ${ }^{22,23}$ Elevated lactate and glycine levels was also been associated with lower survival rates in ER positive BC patients receiving surgery as primary treatment. ${ }^{60}$ Furthermore, lactate has been associated with poor prognosis in other cancers and is a generally accepted marker for tumor aggressiveness, as high levels of lactate have been correlated to low survival rates, high incidence of distant metastasis and recurrence. ${ }^{61,} 62$ Increased lactate production and rapid glucose consumption are known characteristics of the Warburg effect, which can be observed in most cancer cells. ${ }^{63}$ Glycine has been linked to cancer-induced metabolic reprogramming, and glycine consumption and expression of the mitochondrial glycine biosynthetic pathway have been identified to be strongly correlated with the rates of proliferation across cancer cells. ${ }^{64}$

The RCB response measure represents an independent prognostic factor of distant relapse-free survival (DRFS) in multivariate Cox regression analyses of cancer patients. ${ }^{29}$ 
RCB 0 and I are associated with good prognosis, while RCB II and III are associated with poor prognosis. Based on serum metabolic profiles, we could not predict patient response to treatment before or during treatment. However, patients with a good prognosis could be discriminated from patients with a poor prognosis six weeks after treatment completion (TP4) with an accuracy of $69 \%$ ( $p$-value $=0.001)$. RCB II or III patients had higher serum levels of citrate and lower levels of phenylalanine and histidine. Significantly higher serum levels of citrate and lower of phenylalanine and histidine have been observed in metabolic profiles of metastatic compared to early $B C$ implying that they play a role in the formation of metastasis. ${ }^{58}$

Patients receiving bevacizumab were significantly discriminated from those treated only with chemotherapy 12 weeks into treatment (TP2). Discriminating metabolites were lower levels of leucine, acetoacetate, and tri-hydroxybutyrate and higher levels of formate in patients receiving bevacizumab. A previous study has linked the rate of $\beta$ hydroxybutyrate and acetoacetate in blood to mitochondrial activity. ${ }^{65}$ The effect of bevacizumab on the serum metabolome of $\mathrm{BC}$ patients has, to our knowledge, not been 
described previously. A study on metastatic renal cell carcinoma identified changes in glucose, $\mathrm{N}$-acetylglycoproteins, lipids and lipoproteins as an effect of treatment, relating these to known side effects of the drugs bevacizumab and temsirolimus. ${ }^{66}$ Our previous study on tissue metabolites from the same patient cohort ${ }^{21}$ showed weak associations between bevacizumab and tissue metabolic profiles.

An advantage of this study cohort is that both tissue biopsies and serum samples were available from the same patients, allowing for a comparison of metabolic information. Importantly, the metabolic information from these two types of biological samples is different, with some significant, but low correlations (Figure 1B). This explains why we could predict patient survival from tissue, but not serum metabolites. Although tumors are often characterized by high lactate production, there was no correlation between tissue and serum lactate levels. A study linking tumor information in early BC patients with plasma metabolites, showed an inverse correlation between plasma lactate levels and the tumor size. ${ }^{67}$ In general, despite possible leakage of metabolites from the cancer tissue into the bloodstream of the host organism, the overall serum metabolism has larger 
variation that may mask these tumor-derived metabolites; thus, metabolites which have been associated with treatment response when analyzing tumor tissue, are not necessarily relevant in the context of serum metabolomics.

Multivariate analysis, taking advantage of the multilevel structure of the data focusing on the within-subject variations resulted in models with high classification accuracy for characterizing the serum metabolic changes from treatment. Our study also pinpoints that awareness regarding the effect of normalization procedures is necessary, given the different results observed with the exclusion of lipid signals prior to a second normalization of the serum metabolic profiles. Although different normalization strategies did not affect the quality of the multivariate models per se, making their robustness evident, variables important for the classifications were affected, making comparisons of potential biomarkers across studies challenging. 


\section{CONCLUSION}

By metabolic profiling of serum sampled before, during and after neoadjuvant treatment in breast cancer patients, we have revealed significant metabolic changes in serum as a response to treatment. This gives an insight into how the body is affected by treatment, and provides a possible tool for understanding negative side-effects of treatment. Serum metabolomics therefore has a potential for longitudinal patient-monitoring during and after breast cancer treatment.

Tissue metabolic profiles during treatment were significantly correlated to five-year survival, while no such information was apparent in the serum metabolic profiles. Importantly, we demonstrate low correlations between serum and tissue metabolites, emphasizing the complementary nature of the metabolic information in these biological matrices. 


\author{
ABBREVIATIONS \\ BC: Breast cancer; BMI: Body Mass Index; CPMG, Carr-Purcell-Meiboom-Gill; CVD: \\ Cardio-Vascular Disease; DRFS, Distant Relapse-Free Survival; ER, Estrogen \\ Receptor; HER, Human Epidermal Growth Factor Receptor; LABC Locally advanced \\ breast cancer; LV, Loading Vector; MR, Magnetic Resonance; NAC: Neoadjuvant \\ chemotherapy; NMR, Nuclear Magnetic Resonance; NOESY, Nuclear Overhauser \\ Effect Spectroscopy; PC, Principal Component; PCA, Principal Component Analysis; \\ pCR, Pathologic Complete Response; PgR, Progesterone Receptor; PLS-DA, Partial \\ Least Squares Discriminant Analysis; RCB, Residual Cancer Burden; TP1, TP2, TP3, \\ TP4, Time points for sampling: Before treatment, 12 weeks into treatment, 25 weeks \\ into treatment, and 6 weeks after treatment, respectively; VIP, Variable Importance in \\ Projection
}

SUPPORTING INFORMATION

The following files are available free of charge at ACS website: http://pubs.acs.org :

Figure S1. Flow diagram showing the experimental set up of the study. 
Figure S2. Sample availability at each sampling time point, including survival data.

Table S1. Details on quantification of serum metabolites.

Figure S3. A representative spectrum with annotated metabolite peaks.

Figure S4. PCA scores plot of the serum metabolites, colored according to the time point at which they have been obtained.

Figure S5. Scores and loading plot of multilevel PLS-DA analyses on serum metabolites with lipid peaks, comparing TP1 with TP4.

Figure S6. Scores and loading plots of multilevel PLS-DA analyses on serum metabolites.

\author{
Notes \\ The NeoAva study was co-sponsored by Roche Norway and Sanofi-Aventis Norway. Oslo \\ University Hospital is the main sponsor for the NeoAva study.
}

ACKNOWLEDGMENT 
The NMR analysis was performed at the MR Core Facility, Norwegian University of

Science and Technology (NTNU), which is funded by the Faculty of Medicine and Health

Sciences at NTNU and the Central Norway Regional Health Authority. Part of this work

was supported by the Norwegian Cancer Society (grant 163243) 


\section{REFERENCES}

(1) Cancer Registry of Norway Cancer in Norway 2017 - Cancer incidence, mortality, survival and prevalence in Norway, 2018.

(2) Sørlie, T.; Perou, C. M.; Tibshirani, R.; Turid Aas, S. G.; Johansen, H.; Hastie, T.; Eisen, M. B.; Rijn, M. v. d.; Jefferey, S. S.; Thorsen, T.; Quist, H.; Matese, J. C.; Brown, P. O.; Botstein, D.; Lønning, P. E.; Børresen-Dale, A.-L., Gene expression patterns of breast carcinomas distinguish tumor subclasses with clinical implications. Proceedings of the National Academy of Sciences of the United States of America 2011, 98 (19).

(3) Sitter, B.; Bathen, T. F.; Singstad, T. E.; Fjøsne, H. E.; Lundgren, S.; Halgunset, J.; Gribbestad, I. S., Quantification of metabolites in breast cancer patients with different clinical prognosis using HR MAS MR spectroscopy. NMR in biomedicine 2009, 23 (4).

(4) Haukaas, T. H.; Euceda, L. R.; Giskeodegard, G. F.; Lamichhane, S.; Krohn, M.; Jernstrom, S.; Aure, M. R.; Lingjaerde, O. C.; Schlichting, E.; Garred, O.; Due, E. U.; Mills, G. B.; Sahlberg, K. K.; Borresen-Dale, A. L.; Bathen, T. F.; Oslo Breast Cancer, C., Metabolic clusters of breast cancer in relation to geneand protein expression subtypes. Cancer Metab 2016, 4, 12.

(5) Aure, M. R.; Vitelli, V.; Jernstrom, S.; Kumar, S.; Krohn, M.; Due, E. U.; Haukaas, T. H.; Leivonen, S. K.; Vollan, H. K.; Luders, T.; Rodland, E.; Vaske, C. J.; Zhao, W.; Moller, E. K.; Nord, S.; Giskeodegard, G. F.; Bathen, T. F.; Caldas, C.; Tramm, T.; Alsner, J.; Overgaard, J.; Geisler, J.; Bukholm, I. R.; Naume, B.; Schlichting, E.; Sauer, T.; Mills, G. B.; Karesen, R.; Maelandsmo, G. M.; Lingjaerde, O. C.; Frigessi, A.; Kristensen, V. N.; Borresen-Dale, A. L.; Sahlberg, K. K.; Osbreac, Integrative clustering reveals a novel split in the luminal A subtype of breast cancer with impact on outcome. Breast Cancer Res 2017, $19(1), 44$.

(6) Zhao, X.; Rodland, E. A.; Tibshirani, R.; Plevritis, S., Molecular subtyping for clinically defined breast cancer subgroups. Breast Cancer Res 2015, 17, 29. 
(7) The Norwegian Cancer Registry Data and statistics; Institute of population-based cancer research: Oslo, 2019.

(8) Makhoul, I.; Kiwan, E., Neoadjuvant systemic treatment of breast cancer. J Surg Oncol 2011, 103 (4), 348-57.

(9) Miller, E.; Lee, H. J.; Lulla, A.; Hernandez, L.; Gokare, P.; Lim, B., Current treatment of early breast cancer: adjuvant and neoadjuvant therapy. F1000Res 2014, 3, 198.

(10) Folkman, J., Tumor angiogenesis: therapeutic implications. N Eng/ J Med 1971, 285(21), 1182-6.

(11) Hanahan D, W. R., Hallmarks of cancer: the next generation. Cel/ 2011, 144 (5), 646-74.

(12) Ranieri, G.; Patruno, R.; Ruggieri, E.; Montemurro, S.; Valerio, P.; Ribatti, D., Vascular endothelial growth factor (VEGF) as a target of bevacizumab in cancer: from the biology to the clinic. Curr Med Chem 2006, 13 (16), 1845-57.

(13) Monneur, A.; Goncalves, A.; Gilabert, M.; Finetti, P.; Tarpin, C.; Zemmour, C.; Extra, J. M.; Tallet, A.; Lambaudie, E.; Jacquemier, J.; Houvenaeghel, G.; Boher, J. M.; Viens, P.; Bertucci, F., Similar response profile to neoadjuvant chemotherapy, but different survival, in inflammatory versus locally advanced breast cancers. Oncotarget 2017, 8 (39), 66019-66032.

(14) Wang, M.; Hou, L.; Chen, M.; Zhou, Y.; Liang, Y.; Wang, S.; Jiang, J.; Zhang, Y., Neoadjuvant Chemotherapy Creates Surgery Opportunities For Inoperable Locally Advanced Breast Cancer. Sci Rep 2017, 7, 44673.

(15) Berger, A. M.; Mooney, K.; Alvarez-Perez, A.; Breitbart, W. S.; Carpenter, K. M.; Cella, D.; Cleeland, C.; Dotan, E.; Eisenberger, M. A.; Escalante, C. P.; Jacobsen, P. B.; Jankowski, C.; LeBlanc, T.; Ligibel, J. A.; Loggers, E. T.; Mandrell, B.; Murphy, B. A.; Palesh, O.; Pirl, W. F.; Plaxe, S. C.; Riba, M. B.; Rugo, H. S.; Salvador, C.; Wagner, L. I.; Wagner-Johnston, N. D.; Zachariah, F. J.; Bergman, M. A.; Smith, C., Cancer-Related Fatigue, Version 2.2015. Journal of the National Comprehensive Cancer Network 2015, 13. 
(16) Bower, J. E.; Ganz, P. A.; Desmond, K. A.; Rowland, J. H.; Meyerowitz, B. E.; Belin, T. R., Fatigue in breast cancer survivors: occurrence, correlates, and impact on quality of life. J Clin Onco/2000, 18(4), 743-53.

(17) Bower, J. E.; Wiley, J.; Petersen, L.; Irwin, M. R.; Cole, S. W.; Ganz, P. A., Fatigue after breast cancer treatment: Biobehavioral predictors of fatigue trajectories. Health Psycho/2018, 37 (11), 1025-1034.

(18) Oh, P.-J.; Cho, J.-R., Changes in Fatigue, Psychological Distress, and Quality of Life After Chemotherapy in Women with Breast Cancer: A Prospective Study. Cancer Nursing 2018, $O(0)$.

(19) Aleman, B. M.; Moser, E. C.; Nuver, J.; Suter, T. M.; Maraldo, M. V.; Specht, L.; Vrieling, C.; Darby, S. C., Cardiovascular disease after cancer therapy. EJC Supp/2014, 12 (1), 18-28.

(20) Markley, J. L.; Bruschweiler, R.; Edison, A. S.; Eghbalnia, H. R.; Powers, R.; Raftery, D.; Wishart, D. S., The future of NMR-based metabolomics. Curr Opin Biotechno/2017, 43, 34-40.

(21) Euceda, L. R.; Haukaas, T. H.; Giskeødegård, G. F.; Vettukattil, R.; Engel, J.; Silwal-Pandit, L.; Lundgren, S.; Borgen, E.; Garred, Ø.; Postma, G.; Buydens, L. M. C.; Børresen-Dale, A.-L.; Engebraaten, O.; Bathen, T. F., Evaluation of metabolomic changes during neoadjuvant chemotherapy combined with bevacizumab in breast cancer using MR spectroscopy. Metabolomics 2017, 13 (4), 37.

(22) Cao, M. D.; Giskeodegard, G. F.; Bathen, T. F.; Sitter, B.; Bofin, A.; Lonning, P. E.; Lundgren, S.; Gribbestad, I. S., Prognostic value of metabolic response in breast cancer patients receiving neoadjuvant chemotherapy. BMC Cancer 2012, 12, 39.

(23) Cao, M. D.; Sitter, B.; Bathen, T. F.; Bofin, A.; Lonning, P. E.; Lundgren, S.; Gribbestad, I. S., Predicting long-term survival and treatment response in breast cancer patients receiving neoadjuvant chemotherapy by MR metabolic profiling. NMR Biomed 2012, 25 (2), 369-78. 
(24) Giskeødegård, G. F. Identification and characterization of prognostic factors in breast cancer using MR metabolomics. Doctoral thesis, Norwegian University of Science and Technology, Trondheim, 2011.

(25) Giskeødegård, G. F.; Madssen, T. S.; Euceda, L. R.; Tessem, M.-B.; Moestue, S. A.; Bathen, T. F., NMR-based metabolomics of biofluids in cancer. NMR Biomedicine 2018, (Special Issue Review Article).

(26) Jabeen, S.; Zucknick, M.; Nome, M.; Dannenfelser, R.; Fleischer, T.; Kumar, S.; Luders, T.; von der Lippe Gythfeldt, H.; Troyanskaya, O.; Kyte, J. A.; Borresen-Dale, A. L.; Naume, B.; Tekpli, X.; Engebraaten, O.; Kristensen, V., Serum cytokine levels in breast cancer patients during neoadjuvant treatment with bevacizumab. Oncoimmunology 2018, 7(11), e1457598.

(27) Hoglander, E. K.; Nord, S.; Wedge, D. C.; Lingjaerde, O. C.; Silwal-Pandit, L.; Gythfeldt, H. V.; Vollan, H. K. M.; Fleischer, T.; Krohn, M.; Schlitchting, E.; Borgen, E.; Garred, O.; Holmen, M. M.; Wist, E.; Naume, B.; Van Loo, P.; Borresen-Dale, A. L.; Engebraaten, O.; Kristensen, V., Time series analysis of neoadjuvant chemotherapy and bevacizumab-treated breast carcinomas reveals a systemic shift in genomic aberrations. Genome Med2018, 10 (1), 92.

(28) Silwal-Pandit, L.; Nord, S.; von der Lippe Gythfeldt, H.; Moller, E. K.; Fleischer, T.; Rodland, E.; Krohn, M.; Borgen, E.; Garred, O.; Olsen, T.; Vu, P.; Skjerven, H.; Fangberget, A.; Holmen, M. M.; Schlitchting, E.; Wille, E.; Nordberg Stokke, M.; Moen Vollan, H. K.; Kristensen, V.; Langerod, A.; Lundgren, S.; Wist, E.; Naume, B.; Lingjaerde, O. C.; Borresen-Dale, A. L.; Engebraaten, O., The Longitudinal Transcriptional Response to Neoadjuvant Chemotherapy with and without Bevacizumab in Breast Cancer. Clin Cancer Res 2017, 23 (16), 4662-4670.

(29) Symmans, W. F.; Peintinger, F.; Hatzis, C.; Rajan, R.; Kuerer, H.; Valero, V.; Assad, L.; Poniecka, A.; Hennessy, B.; Green, M.; Buzdar, A. U.; Singletary, S. E.; Hortobagyi, G. N.; Pusztai, L., Measurement of residual breast cancer burden to predict survival after neoadjuvant chemotherapy. J Clin Onco/2007, 25 (28), 4414-22.

(30) MATLAB, R2017b; The MathWorks Inc.: Natick, Massachusetts, 2017. 
(31) Tomasi, G.; Savorani, F.; Engelsen, S. B., icoshift: An effective tool for the alignment of chromatographic data. Journal of chromatography. A 2011, 1218 (43), 7832-40.

(32) Cloarec, O.; Dumas, M. E.; Craig, A.; Barton, R. H.; Trygg, J.; Hudson, J.; Blancher, C.; Gauguier, D.; Lindon, J. C.; Holmes, E.; Nicholson, J., Statistical total correlation spectroscopy: an exploratory approach for latent biomarker identification from metabolic 1H NMR data sets. Anal Chem 2005, 77 (5), 1282-9.

(33) Aru, V.; Lamb, C.; Khakimov, B.; C.J.Hoefsloot, H.; Zwanenburg, G.; Lind, M. V.; Schäfer, H.; Duynhovende, J.; M.Jacobs, D.; K.Smilde, A.; B.Engelsen, S., Quantification of lipoprotein profiles by nuclear magnetic resonance spectroscopy and multivariate data analysis. TrAC Trends in Analytical Chemistry 2017, 94, 210-219.

(34) Dieterle, F.; Ross, A.; Schlotterbeck, G.; Senn, H., Probabilistic quotient normalization as robust method to account for dilution of complex biological mixtures. Application in 1H NMR metabonomics. Anal Chem 2006, 78(13), 4281-90.

(35) Wold, S.; Esbensen, K.; Geladi, P., Principal Component Analysis. Chemometrics and Intelligent Laboratory Systems 1987, 2, 37-52.

(36) Wold, S.; Sjöström, M.; Eriksson, L., PLS-regression: a basic tool of chemometrics. Chemometrics and Intelligent Laboratory Systems 2001, 58 (2), 109-130.

(37) Westerhuis, J. A.; Hoefsloot, H. C. J.; Smit, S.; Vis, D. J.; Smilde, A. K.; van Velzen, E. J. J.; van Duijnhoven, J. P. M.; van Dorsten, F. A., Assessment of PLSDA cross validation. Metabolomics 2008, 4(1), 81-89.

(38) Westerhuis, J. A.; van Velzen, E. J.; Hoefsloot, H. C.; Smilde, A. K., Multivariate paired data analysis: multilevel PLSDA versus OPLSDA. Metabolomics 2010, 6 (1), 119-128.

(39) Eigenvector Research, I. PLS Toolbox, 8.6.2; Manson, WA USA 98831, 2018.

(40) Mehmood, T.; Liland, K. H.; Snipen, L.; Sæbø, S., A review of variable selection methods in Partial Least Squares Regression. Chemometrics and Intelligent Laboratory Systems 2012, 118, 62-69. 
(41) Rosner, B., Fundamentals of Biostatistics. Eight ed.; Cengage Learning: Boston, 2015.

(42) Benjamini, Y.; Hockberg, Y., Controlling the false discobery rate: A practical and powerful approach for multiple testing. Journal of the Royal Statistical Society $B$ 1995, 57, 289-300.

(43) Wei, T.; Simko, V. R package "corrplot": Visualization of a Correlation Matrix, 2017.

(44) R Development Core Team R: A Language and Environment for Statistical Computing, Vienna, Austria, 2009.

(45) Yu, Z.; Zhai, G.; Singmann, P.; He, Y.; Xu, T.; Prehn, C.; Romisch-Margl, W.; Lattka, E.; Gieger, C.; Soranzo, N.; Heinrich, J.; Standl, M.; Thiering, E.; Mittelstrass, K.; Wichmann, H. E.; Peters, A.; Suhre, K.; Li, Y.; Adamski, J.; Spector, T. D.; Illig, T.; Wang-Sattler, R., Human serum metabolic profiles are age dependent. Aging Cel/2012, 11 (6), 960-7.

(46) Esko, T.; Hirschhorn, J. N.; Feldman, H. A.; Hsu, Y. H.; Deik, A. A.; Clish, C. B.; Ebbeling, C. B.; Ludwig, D. S., Metabolomic profiles as reliable biomarkers of dietary composition. Am J Clin Nutr 2017, 105 (3), 547-554.

(47) Sato, S.; Parr, E. B.; Devlin, B. L.; Hawley, J. A.; Sassone-Corsi, P., Human metabolomics reveal daily variations under nutritional challenges specific to serum and skeletal muscle. Mol Metab 2018, 16, 1-11.

(48) Jove, M.; Collado, R.; Quiles, J. L.; Ramirez-Tortosa, M. C.; Sol, J.; RuizSanjuan, M.; Fernandez, M.; de la Torre Cabrera, C.; Ramirez-Tortosa, C.; Granados-Principal, S.; Sanchez-Rovira, P.; Pamplona, R., A plasma metabolomic signature discloses human breast cancer. Oncotarget 2017, 8 (12), 19522-19533.

(49) Cala, M. P.; Aldana, J.; Medina, J.; Sanchez, J.; Guio, J.; Wist, J.; Meesters, R. J. W., Multiplatform plasma metabolic and lipid fingerprinting of breast cancer: A pilot control-case study in Colombian Hispanic women. PLoS One 2018, 13 (2), e0190958. 
(50) Xie, G.; Zhou, B.; Zhao, A.; Qiu, Y.; Zhao, X.; Garmire, L.; Shvetsov, Y. B.; $\mathrm{Yu}, \mathrm{H}$.; Yen, Y.; Jia, W., Lowered circulating aspartate is a metabolic feature of human breast cancer. Oncotarget 2015, 6(32), 33369-81.

(51) Quu, Y.; Zhou, B.; Su, M.; Baxter, S.; Zheng, X.; Zhao, X.; Yen, Y.; Jia, W., Mass spectrometry-based quantitative metabolomics revealed a distinct lipid profile in breast cancer patients. Int J Mol Sci 2013, 14 (4), 8047-61.

(52) Gu, H.; Pan, Z.; Xi, B.; Asiago, V.; Musselman, B.; Raftery, D., Principal component directed partial least squares analysis for combining nuclear magnetic resonance and mass spectrometry data in metabolomics: application to the detection of breast cancer. Analytica chimica acta 2011, 686 (1-2), 57-63.

(53) Jobard, E.; Tredan, O.; Bachelot, T.; Vigneron, A. M.; Ait-Oukhatar, C. M.; Arnedos, M.; Rios, M.; Bonneterre, J.; Dieras, V.; Jimenez, M.; Merlin, J. L.; Campone, M.; Elena-Herrmann, B., Longitudinal serum metabolomics evaluation of trastuzumab and everolimus combination as pre-operative treatment for HER2 positive breast cancer patients. Oncotarget 2017, 8 (48), 83570-83584.

(54) Wei, S.; Liu, L.; Zhang, J.; Bowers, J.; Gowda, G. A.; Seeger, H.; Fehm, T.; Neubauer, H. J.; Vogel, U.; Clare, S. E.; Raftery, D., Metabolomics approach for predicting response to neoadjuvant chemotherapy for breast cancer. $\mathrm{Mol} O \mathrm{Oncol}$ 2013, 7(3), 297-307.

(55) Jiang, L.; Lee, S. C.; Ng, T. C., Pharmacometabonomics Analysis Reveals Serum Formate and Acetate Potentially Associated with Varying Response to Gemcitabine-Carboplatin Chemotherapy in Metastatic Breast Cancer Patients. J Proteome Res 2018, 17 (3), 1248-1257.

(56) Madssen, T. S.; Thune, I.; Flote, V. G.; Lundgren, S.; Bertheussen, G. F.; Frydenberg, H.; Wist, E.; Schlichting, E.; Schafer, H.; Fjosne, H. E.; Vettukattil, R.; Lomo, J.; Bathen, T. F.; Giskeodegard, G. F., Metabolite and lipoprotein responses and prediction of weight gain during breast cancer treatment. Br J Cancer 2018, 119 (9), 1144-1154.

(57) Tenori, L.; Oakman, C.; Morris, P. G.; Gralka, E.; Turner, N.; Cappadona, S.; Fornier, M.; Hudis, C.; Norton, L.; Luchinat, C.; Di Leo, A., Serum metabolomic profiles evaluated after surgery may identify patients with oestrogen receptor 
negative early breast cancer at increased risk of disease recurrence. Results from a retrospective study. Mol Onco/2015, 9(1), 128-39.

(58) Hart, C. D.; Vignoli, A.; Tenori, L.; Uy, G. L.; Van To, T.; Adebamowo, C.; Hossain, S. M.; Biganzoli, L.; Risi, E.; Love, R. R.; Luchinat, C.; Di Leo, A., Serum Metabolomic Profiles Identify ER-Positive Early Breast Cancer Patients at Increased Risk of Disease Recurrence in a Multicenter Population. Clin Cancer Res 2017, 23 (6), 1422-1431.

(59) Li, X.; Sun, L.; Zhang, W.; Li, H.; Wang, S.; Mu, H.; Zhou, Q.; Zhang, Y.; Tang, Y.; Wang, Y.; Chen, W.; Yang, R.; Dong, J., Association of serum glycine levels with metabolic syndrome in an elderly Chinese population. Nutr Metab (Lond) 2018, 15, 89.

(60) Giskeodegard, G. F.; Lundgren, S.; Sitter, B.; Fjosne, H. E.; Postma, G.; Buydens, L. M.; Gribbestad, I. S.; Bathen, T. F., Lactate and glycine-potential MR biomarkers of prognosis in estrogen receptor-positive breast cancers. NMR Biomed 2012, 25(11), 1271-9.

(61) Walenta, S.; Mueller-Klieser, W. F., Lactate: mirror and motor of tumor malignancy. Semin Radiat Oncol 2004, 14 (3), 267-74.

(62) Walenta, S.; Wetterling, M.; Lehrke, M.; Schwickert, G.; Sundfor, K.; Rofstad, E. K.; Mueller-Klieser, W., High lactate levels predict likelihood of metastases, tumor recurrence, and restricted patient survival in human cervical cancers. Cancer Res 2000, 60 (4), 916-21.

(63) Vander Heiden, M. G.; Cantley, L. C.; Thompson, C. B., Understanding the Warburg effect: the metabolic requirements of cell proliferation. Science 2009, 324 (5930), 1029-33.

(64) Jain, M.; Nilsson, R.; Sharma, S.; Madhusudhan, N.; Kitami, T.; Souza, A. L.; Kafri, R.; Kirschner, M. W.; Clish, C. B.; Mootha, V. K., Metabolite profiling identifies a key role for glycine in rapid cancer cell proliferation. Science 2012, 336 (6084), 1040-4.

(65) Galan, A.; Hernandez, J.; Jimenez, O., Measurement of blood acetoacetate and beta-hydroxybutyrate in an automatic analyser. J Autom Methods Manag Chem 2001, 23 (3), 69-76. 
(66) Jobard, E.; Blanc, E.; Négrier, S.; Escudier, B.; Gravis, G.; Chevreau, C.; Elena-Herrmann, B.; Trédan, O., A serum metabolomic fingerprint of bevacizumab and temsirolimus combination as first-line treatment of metastatic renal cell carcinoma. British Journal of Cancer 2015, 113, 1148-1157.

(67) Richard, V.; Conotte, R.; Mayne, D.; Colet, J. M., Does the 1H-NMR plasma metabolome reflect the host-tumor interactions in human breast cancer? Oncotarget 2017, 8 (30), 49915-49930. 


\section{For Table of Contents Only}

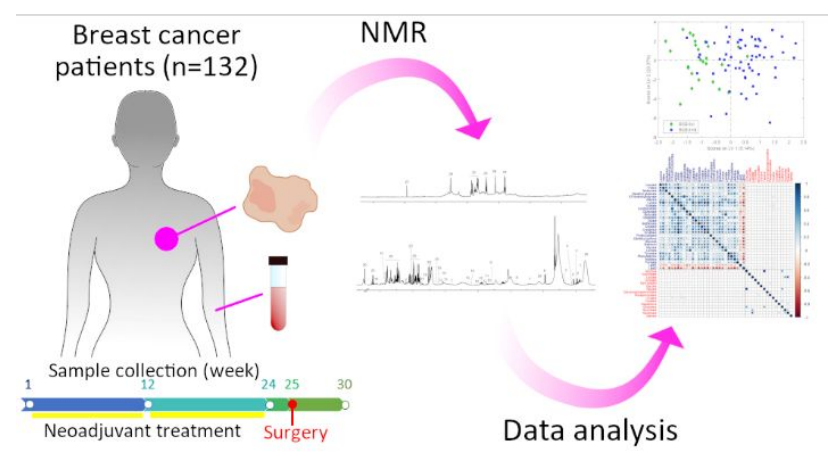

